\author{
ANALIZA COMPARATIVĂ A DISTRIBUTIEI PRESIUNILOR PLANTARE ÎN DINAMICĂ PE DIFERITE ZONE ALE \\ PICIORULUI
}

\author{
Mariana COSTEA, Aura MIHAI*
}

Gheorghe Asachi Technical University of lasi, 67 Dimitrie Mangeron Blvd., lasi, Romania, amihai@tex.tuiasi.ro

\title{
COMPARATIVE ANALYSIS OF DYNAMIC PLANTAR PRESSURE DISTRIBUTION ON DIFFERENT AREAS OF THE FOOT
}

ABSTRACT. The plantar distribution between the foot and the contact surface provides valuable information about the foot's structure and functions. The plantar footprints were taken from 60 young women, aged between 20-30 and their foot typology was established by the authors in a previous study. The average values of the plantar pressures recorded on ten foot segments, in case of the four groups: normal foot, high arched foot, flat foot and Hallux-Valgus foot were analyzed in the present study. By comparing the pressures for the four identified groups, it was demonstrated that there are no significant differences between subjects, the maximum values being registered on the third metatarsal head bone, followed by the second metatarsal head bone and the heel. The findings of a Pearson correlation analysis demonstrate that some pressure areas are directly proportional to others (like for example toe areas with first, second, fourth metatarsal head bones and median area of the foot) and other pressure areas are indirectly proportional to others (like for example lateral heel, with second to fifth toe). An important result of the performed analysis is the negative correlation between toe area and the third and fifth metatarsals head bones. Therefore, when creating the footwear bottom components, the designer should use different materials and structures and design in order to redistribute the plantar pressures according to areas of the foot.

KEYWORDS: plantar footprint, gait, pressure areas, footwear

ANALIZA COMPARATIVĂ A DISTRIBUTIEI PRESIUNILOR PLANTARE ÎN DINAMICĂ PE DIFERITE ZONE ALE PICIORULUI

REZUMAT. Distribuţia presiunii manifestate între talpa piciorului şi planul de sprijin furnizează informaţii valoroase privind structura şi funcţiile piciorului. Într-un studiu anterior autorii au preluat amprentele plantare la un număr de 60 femei, cu vârsta cuprinsă între 20-30 de ani şi au stabilit tipologia piciorului acestora. În cadrul prezentului studiu au fost comparate valorile medii ale presiunilor pe zece segmente al suprafetei plantare, pentru fiecare din cele 4 grupe: picior normal, picior scobit, picior plat şi picior cu Hallux-Valgus, identificate de autori într-un studiu anterior. Comparând presiunile pentru cele 4 grupe de subiecţi s-a observat că nu există diferenţe semnificative între aceştia, presiunea maximă înregistrându-se de fiecare dată pe metatarsianul al treilea, urmat de metatarsianul al doilea şi călcâi. Printr-o analiză statistică folosind coeficientul de corelaţie Pearson s-a demonstrat că anumite zone de presiune sunt direct proporţionale cu altele (de exemplu zona degetelor cu primul, al doilea și al patrulea metatarsian, precum şi cu zona mediană a piciorului) și alte zone de presiune sunt indirect proporţionale cu altele (de exemplu zona exterioară a călcâiului cu degetul cinci). Un rezultat important al acestei analize statistice este corelaţia negativă identificată între zona degetelor şi al treilea şi al cincilea metatarsian. În consecinţă, în momentul proiectării ansamblului inferior al încălţămintei, proiectantul trebuie să aibă în vedere echilibrarea distribuţiei presiunilor plantare pe zonele piciorului, prin forma şi structura modelului şi prin tipul materialelor folosite.

CUVINTE CHEIE: amprentă plantară, mers, zone de presiune, încălţăminte

LA COMPARAISON DE LA RÉPARTITION DES PRESSIONS PLANTAIRES EN DYNAMIQUE DANS DE DIFFERENTES ZONES DU PIED

RÉSUMÉ. La répartition de la pression existante entre le pied et la surface de contact fournisse des informations précieuses sur la structure et les fonction du pied. Dans une précédente étude, les auteurs ont enregistré les empreintes plantaires d'un nombre de 60 femmes, âgées de 20-30 ans, et ont établi leur typologie du pied. Dans la présente étude on a comparé les valeurs moyennes de la pression sur les dix segments de la surface plantaire, pour chacun des 4 groupes: pied normal, pied haut-arqué, pied plat et l'hallux valgus, identifiés dans une étude précédente. Après la comparaison des pressions pour les 4 groupes de sujets a été observé qu'il n'y a pas de différences significatives entre les sujets, la pression maximale a été enregistrée chaque fois sur le troisième métatarse, suivi par le deuxième métatarsien et le talon. Par une analyse statistique en utilisant le coefficient de corrélation de Pearson on a constaté que certaines zones de la pression sont directement proportionnelles entre elles (par exemple la zone des orteils avec le première, le deuxième et le quatrième métatarsien et avec la zone médiane du pied) et d'autres zones de pression sont indirectement proportionnelles à d'autres (par exemple, la zone externe du talon avec le cinquième orteil). Un résultat important de cette analyse statistique est la corrélation négative identifiée entre la zone des orteils et le troisième et le cinquième métatarsien. Par conséquent, lors de la conception de l'ensemble inférieur de chaussures, le concepteur doit envisager d'équilibrer la répartition des pressions plantaires sur les zones du pied, par la forme et la structure du modèle et le type de matériaux utilisés.

MOTS-CLÉS: empreinte plantaire, marche, zones de pression, chaussures

\section{INTRODUCTION}

Walking is a complex motor skill related to the body separation from the bearing plane and its movement. Biomechanical studies have progressed by comparing the presence or the absence of the foot typology parameters between groups with or without

\section{INTRODUCERE}

Mersul este acea deprindere motorie complexă legată de desprinderea corpului de planul de reazem şi deplasarea lui în spaţiu, în poziţie verticală. Studiile de biomecanică au progresat, prin compararea prezenţei, absenţei sau mărimii parametrilor tipologici ai piciorului între grupuri cu sau fără

\footnotetext{
Correspondence to: Aura MIHAl, Gheorghe Asachi Technical University of Iasi, 67 Dimitrie Mangeron Blvd., Iasi, Romania, amihai@tex.tuiasi.ro
} 
pathologies under investigation [1-3].

The plantar pressure represents the forces' distribution on the plantar surface. Plantar pressure measurement gives an indication on the function of the foot and ankle while walking or while performing other physiological activities [4-7]. These data are very important in assessing subjects with abnormal foot or injuries, in the diagnosis and treatment of various diseases associated to musculoskeletal, nervous system and skin [8-10]. Because when walking every step is slightly different from each other due to differences in gait parameters, such as heel contact, speed, strength and effort, there is a change in human locomotion for each individual, even while walking down the same type of ground [11-13].

The evaluation of subjects' pressures could reflect a systemic or localized pathology in the leg and can be indicators (risk factors) or predictors for the development or aggravation of certain pathologies. Atypical plantar pressure values may indicate the need to amend the patient's lifestyle: footwear change, recommendation of orthotics [14].

\section{METHOD}

\section{Equipment}

In order to obtain the plantar footprints, RSscan pressure plate [15], Figure 1 and the associated system, Footscan 7 Gait, 2nd Generation, 0,5 Gait Scientific System, produced by RSscan International were used.

Measuring plantar pressures has become an important tool in analyzing the gait cycle. Plantar pressure distribution between foot and plantar support provides valuable information on the structure and function of the foot, such as, for example, plantar footprint variation in different phases of gait (Figure 1). patologii aflate sub investigare [1-3].

Presiunea plantară reprezintă distribuţia forţelor de apăsare pe suprafaţa plantară. Măsurarea presiunii plantare oferă indicaţii referitoare la funcţiile piciorului şi ale gleznei în ortostatism, în timpul mersului şi al altor activităţi fiziologice [4-7]. Aceste date sunt foarte importante în evaluarea subiecţilor cu anomalii ale picioarelor, dar şi în diagnosticarea şi tratarea altor leziuni asociate cu diferite boli ale aparatului locomotor, ale sistemului nervos şi ale tegumentelor [8-10]. Deoarece în timpul mersului fiecare păşeşte uşor diferit de celălalt, din cauza diferenţelor parametrilor de mers, cum ar fi contactul călcâiului, viteza şi unghiul de înclinare, forţa şi efortul depus, variaţia în locomoţia umană există pentru fiecare individ, chiar în condiţii de mers pe jos pe acelaşi tip de sol [11-13].

În momentul evaluării subiecţilor, presiunile sau şabloanele de distribuţie a presiunilor atipice pot reflecta o patologie sistemică sau localizată la membrul inferior şi pot fi indicatori (factori de risc) sau predictori pentru dezvoltarea sau agravarea unei anumite patologii. Valorile atipice ale presiunii plantare pot indica necesitatea modificării regimului de viaţă a pacientului: schimbarea încălţămintei, indicarea unor orteze [14].

\section{METODĂ}

\section{Echipament}

La obţinerea amprentei plantare a fost folosită placa de presiuni RSscan [15], Figura 1, şi sistemul asociat, Footscan 7 Gait, $2^{\text {nd }}$ Generation, 0,5 Gait Scientific System, producător: RSscan International.

Măsurarea presiunilor plantare a devenit un instrument important în analiza ciclului de mers. Distribuţia presiunii manifestate între talpa piciorului şi planul de sprijin furnizează informaţii valoroase privind structura şi funcţiile piciorului, cum ar fi, de exemplu, variaţia amprentei plantare în diferite fazealemersului (Figura 1).
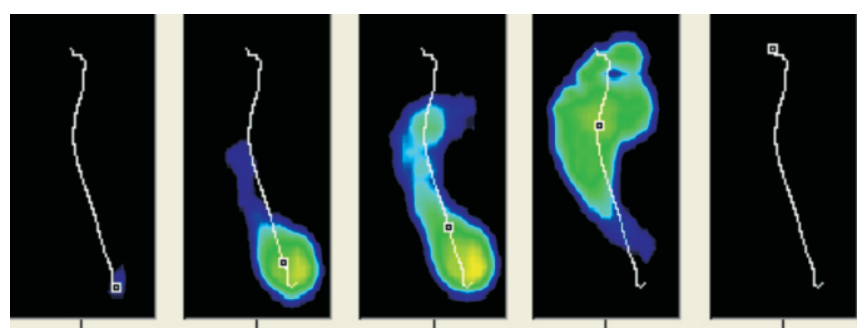

Figure 1. Plantar footprint variation in different phases of gait

Figura 1. Variaţia amprentei plantare în diferite faze ale mersului 


\section{Subjects}

To establish foot typology, the plantar pressures were taken from 60 young women, aged between 20 30 , weighing $45-70 \mathrm{~kg}$. All subjects read and signed an informed consent before testing.

For all investigated subjects the following hypotheses were considered:

- no abnormal walking patterns;

- no structural abnormalities in the foot;

- no systemic diseases that may influence the gait.

\section{RESULTS}

The pressures were taken barefoot. For static measurements, the subjects were required to find their balance on the pressure plate having their weight equally distributed on both feet. For dynamic measurements, the subjects had to walk on pressure plate. Three measurements have been taken in order to obtain reliable and comparable sets of data [16]. The plantar footprint was divided in 10 areas, each of them being differently coloured (Figure 2a). The same legend of colours is kept for pressure graphs, in order to easily recognize how the pressure modifies for each foot area [17] (Figure 2b).

\section{Subiecţi}

În vederea stabilirii tipologiei piciorului, au fost preluate amprentele plantare la un număr de 60 femei, cu vârsta cuprinsă între 20-30 de ani, iar greutatea între 45-70 kg. Toţi subiecţii au citit şi semnat un acord de consimţământ înainte de testare.

Pentru toţi subiecţii investigaţi s-au considerat următoarele ipoteze de lucru:

- nu au fost observate tipare de mers anormale;

- nu prezintă anomalii structurale la nivelul piciorului;

- nu prezintă afecţiuni sistemice care pot influenţa mersul.

\section{REZULTATE}

Presiunile au fost preluate cu piciorul desculţ. Pentru măsurătorile în statică, subiecţii au fost rugaţi să stea cu ambele picioare pe placa de presiuni, într-o poziţie de echilibru. Pentru măsurătorile în dinamică, subiecţii au fost rugaţi să meargă normal pe placă, fiind înregistrată o măsurătoare pentru piciorul drept şi una pentru cel stâng. S-au efectuat câte trei măsurători pentru fiecare subiect pentru a fi obţinute seturi de date fiabile şi comparabile [16]. Talpa piciorului a fost împărţită în 10 zone semnificative (Figura 2a). Aceeaşi legendă a culorilor este păstrată şi la graficele de presiune pentru a putea fi recunoscut mai uşor modul în care presiunile se modifică pe fiecare zonă a piciorului [17] (Figura 2b).

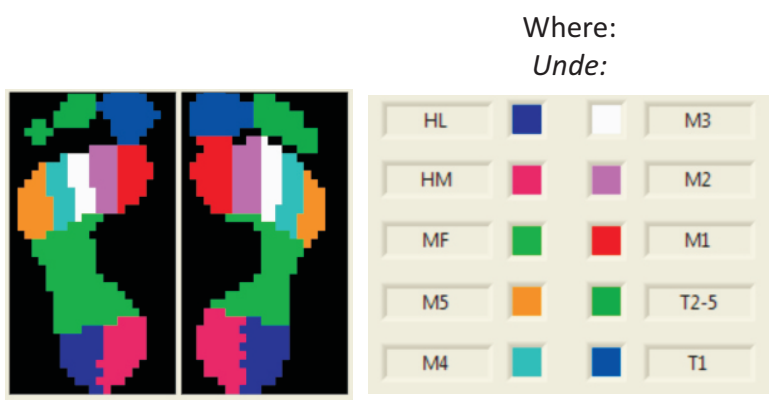

$\mathrm{HL}-$ Lateral Heel
HL - Exterior călcâi
HM -Medial Heel
HM - Interior călcâi
MF - Midfoot
MF-Mijlocul piciorului
M1 - Metatarsal 1
M1-Metatarsian 1
M2 - Metatarsal 2
M2 - Metatarsian 2

M3 - Metatarsal 3

M3 - Metatarsian 3

M4 - Metatarsal 4

M4 - Metatarsian 4

M5 - Metatarsal 5

M5 - Metatarsian 5

T2-5 - Toes 2-5

T2-5 - Degete 2-5

$\mathrm{T} 1$ - Toe 1

T1 - Deget 1

Figura 2a. Foot areas and legend of codes and colours

Figura 2a. Zonele amprentei plantare şi legenda codurilor şi a culorilor
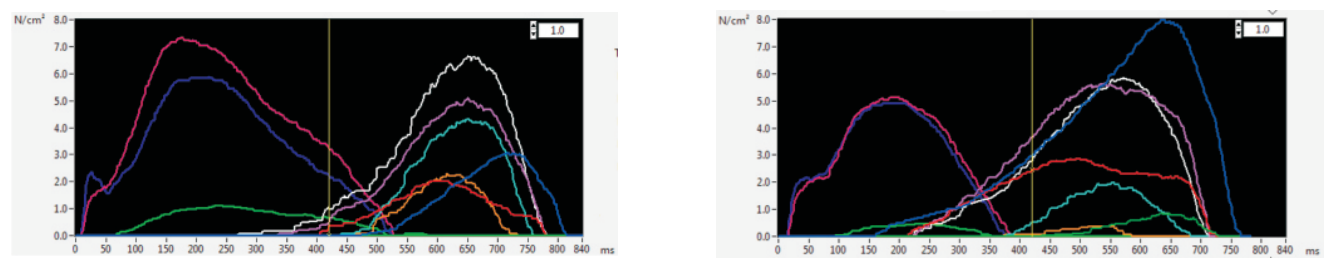

Figure $2 \mathrm{~b}$. Example of pressure graphs depending on the foot areas

Figura $2 \mathrm{~b}$. Exemple de grafice de presiune pe zonele piciorului 


\section{Comparative Analysis of Plantar Pressures}

In a previous study [18], the authors have determined the foot type in case of this specific group, based on plantar footprints. Four types were obtained: normal foot, high arched foot, flat foot and Hallux-Valgus foot. In case of this particular study, the plantar pressures for each of the 10 foot areas were compared for the identified groups. The results are presented in the graphs of Figure 3.

\section{Analiza comparativă a presiunilor plantare}

Într-un studiu anterior [18], autorii au grupat subiecţii acestui grup în funcţie de parametrii amprentei plantare. Au rezultat patru grupe: picior normal, picior scobit, picior plat şi picior cu Hallux-Valgus. În cadrul prezentului studiu, au fost comparate valorile medii ale presiunilor pe fiecare din cele 10 segmente ale suprafeţei plantare pentru grupurile identificate. Rezultatele sunt prezentate în graficele din Figura 3.
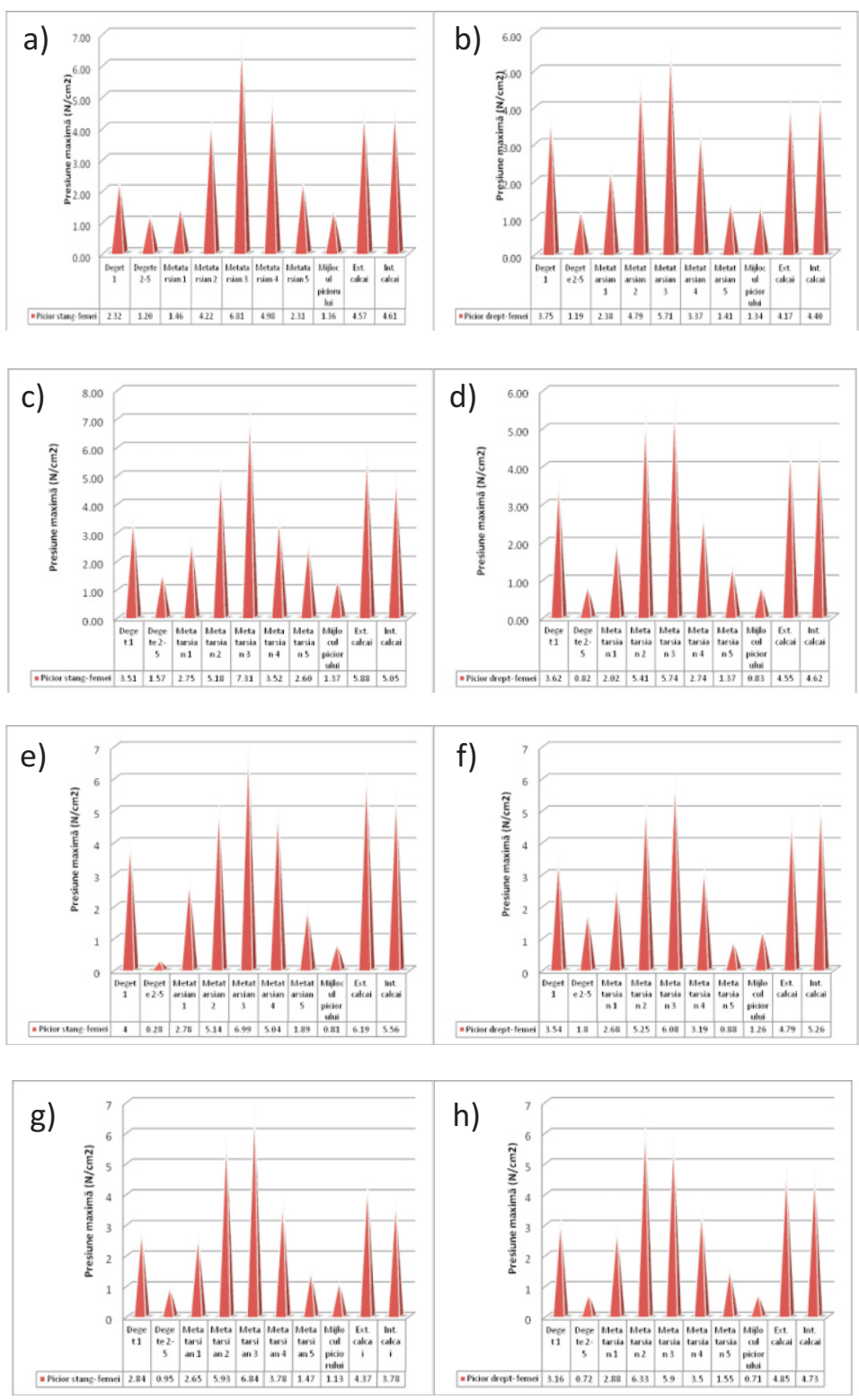

Figure 3. Plantar pressures distribution for left foot: a) normal, c) high arched, e) flat, g) Hallux-Valgus; and for the right foot: b) normal, d) high arched, f) flat, h) Hallux-Valgus

Figura 3. Distribuţia presiunilor plantare pe piciorul stâng: a) normal, c) scobit, e) plat, g) cu Hallux-Valgus, şi pe piciorul drept: b) normal, d) scobit, f) plat, h) cu Hallux-Valgus 
By comparing the pressures for the four identified groups, it was demonstrated that there are no significant differences between subjects, the maximum values being registered on the third metatarsal head bone, followed by the second metatarsal head bone and the heel.

\section{Statistical Analysis}

Using pressure values and SPSS software, a descriptive statistics is performed, Table 1.
Prin compararea presiunilor la cele patru grupuri, s-a demonstrat că nu există diferenţe semnificative între grupurile de subiecţi, presiunea maximă înregistrându-se de fiecare dată pe metatarsianul al treilea, iar următoarele, ca şi valori, pe metatarsianul al doilea şi pe călcâi.

\section{Analiza statistică}

A fost realizată o analiză statistică folosind valorile presiunilor şi aplicaţia software SPSS, Tabelul 1.

Table 1: Descriptive Statistics

Tabelul 1: Analiză statistică descriptivă

\begin{tabular}{|c|c|c|c|c|c|}
\hline & $\begin{array}{c}\text { Number of parameters } \\
\text { Număr parametri }\end{array}$ & $\begin{array}{c}\text { Minimum } \\
\text { Minim }\end{array}$ & $\begin{array}{c}\text { Maximum } \\
\text { Maxim }\end{array}$ & $\begin{array}{c}\text { Mean } \\
\text { Medie }\end{array}$ & $\begin{array}{c}\text { Standard Deviation } \\
\text { Deviaţia standard }\end{array}$ \\
\hline T1 & 60 & 0.00 & 30.86 & 5.8899 & 7.82574 \\
\hline T2-5 & 60 & 0.00 & 60.00 & 6.8821 & 16.96718 \\
\hline M1 & 60 & 0.00 & 43.50 & 5.3396 & 10.02941 \\
\hline M2 & 60 & 0.80 & 92.00 & 13.5739 & 25.15176 \\
\hline M3 & 60 & -19.00 & 21.50 & 6.4515 & 4.95691 \\
\hline M4 & 60 & 0.60 & 47.30 & 7.1664 & 10.94494 \\
\hline M5 & 60 & 0.00 & 19.00 & 1.8933 & 2.64918 \\
\hline MF & 60 & 0.00 & 3.50 & 1.2092 & 0.83466 \\
\hline LH & 60 & 0.40 & 9.10 & 4.9333 & 1.98271 \\
\hline MH & 60 & 1.80 & 8.65 & 4.7608 & 1.61946 \\
\hline Valid N & 60 & & & & \\
\hline Număr valid & & & & & \\
\hline
\end{tabular}

The statistically significant connections between parameters were studied using Pearson correlation.

Pearson's correlation coefficient is the covariance of the two variables divided by the product of their standard deviations [19].
Legăturile semnificative din punct de vedere statistic dintre parametri au fost studiate folosind corelaţia Pearson.

Coeficientul de corelaţie Pearson este covarianţa dintre două variabile împărţită la produsul deviaţiilor lor standard [19].

$$
\mathrm{P}_{\mathrm{X}, \mathrm{Y}}=\frac{\operatorname{cov}(X, Y)}{\sigma \mathrm{Xov}}[20]
$$

where:

- cov is the covariance;

- $\sigma X$ is the standard deviation of $X_{\text {; }}$

- $\sigma \mathrm{Y}$ is the standard deviation of $\mathrm{Y}$.

The results are available in Table 2 . unde:

- cov este covarianţa;

- $\sigma$ X este deviaţia standard a lui X;

- $\sigma$ Y este deviaţia standard a lui Y.

Rezultatele sunt prezentate în Tabelul 2. 
Table 2: Correlations

Tabelul 2: Corelaţii

\begin{tabular}{|c|c|c|c|c|c|c|c|c|c|c|c|}
\hline & & $\mathrm{T} 1$ & $\mathrm{~T} 2-5$ & M1 & M2 & M3 & M4 & M5 & MF & LH & $\mathrm{MH}$ \\
\hline \multirow{3}{*}{ T1 } & $\begin{array}{c}\text { Pearson Correlation } \\
\text { Corelaţia Pearson }\end{array}$ & 1 & $0.955^{* *}$ & $0.893^{* *}$ & $0.964^{* *}$ & -0.029 & $0.869^{* *}$ & -0.190 & -0.153 & $0.417^{* *}$ & 0.172 \\
\hline & Sig. (2-tailed) & & 0.000 & 0.000 & 0.000 & 0.816 & 0.000 & 0.145 & 0.244 & 0.001 & 0.188 \\
\hline & $N$ & 60 & 60 & 60 & 60 & 60 & 60 & 60 & 60 & 60 & 60 \\
\hline \multirow{3}{*}{ T2-5 } & $\begin{array}{c}\text { Pearson Correlation } \\
\text { Corelaţia Pearson }\end{array}$ & $0.955^{* *}$ & 1 & $0.889^{* *}$ & $0.986^{* *}$ & -0.038 & $0.890^{* *}$ & -0.217 & $0.635^{* *}$ & $-0.456^{* *}$ & $-0.365^{* *}$ \\
\hline & Sig. (2-tailed) & 0.000 & & 0.000 & 0.000 & 0.762 & 0.000 & 0.095 & 0.000 & 0.000 & 0.004 \\
\hline & $\mathrm{N}$ & 60 & 60 & 60 & 60 & 60 & 60 & 60 & 60 & 60 & 60 \\
\hline \multirow{3}{*}{ M1 } & $\begin{array}{c}\text { Pearson Correlation } \\
\text { Corelaţia Pearson }\end{array}$ & $0.893^{* *}$ & $0.889^{* *}$ & 1 & $0.932^{* *}$ & 0.038 & $0.967^{* *}$ & -0.160 & $-0.266^{*}$ & $0.405^{* *}$ & 0.225 \\
\hline & Sig. (2-tailed) & 0.000 & 0.000 & & 0.000 & 0.761 & 0.000 & 0.221 & 0.040 & 0.001 & 0.084 \\
\hline & $\mathrm{N}$ & 60 & 60 & 60 & 60 & 60 & 60 & 60 & 60 & 60 & 60 \\
\hline \multirow{3}{*}{ M2 } & $\begin{array}{l}\text { Pearson Correlation } \\
\text { Corelaţia Pearson }\end{array}$ & $0.964^{* *}$ & $0.986^{* *}$ & $0.932^{* *}$ & 1 & 0.028 & $0.927^{* *}$ & 0.166 & $-0.324^{*}$ & $0.572^{* *}$ & $0.450^{* *}$ \\
\hline & Sig. (2-tailed) & 0.000 & 0.000 & 0.000 & & 0.819 & 0.000 & 0.205 & 0.011 & 0.000 & 0.000 \\
\hline & $\mathrm{N}$ & 60 & 60 & 60 & 60 & 60 & 60 & 60 & 60 & 60 & 60 \\
\hline \multirow{3}{*}{$\mathrm{M} 3$} & $\begin{array}{l}\text { Pearson Correlation } \\
\text { Corelaţia Pearson }\end{array}$ & -0.029 & -0.038 & 0.038 & 0.028 & 1 & 0.063 & $0.265^{*}$ & -0.219 & $0.661^{* *}$ & $0.534^{* *}$ \\
\hline & Sig. (2-tailed) & 0.816 & 0.762 & 0.761 & 0.819 & & 0.614 & 0.040 & 0.093 & 0.000 & 0.000 \\
\hline & $\mathrm{N}$ & 60 & 60 & 60 & 60 & 60 & 60 & 60 & 60 & 60 & 60 \\
\hline \multirow{3}{*}{ M4 } & $\begin{array}{l}\text { Pearson Correlation } \\
\text { Corelaţia Pearson }\end{array}$ & $0.869^{* *}$ & $0.890^{* *}$ & $0.967^{* *}$ & $0.927^{* *}$ & 0.063 & 1 & $0.415^{* *}$ & -0.127 & $0.373^{* *}$ & $0.371^{* *}$ \\
\hline & Sig. (2-tailed) & 0.000 & 0.000 & 0.000 & 0.000 & 0.614 & & 0.001 & 0.332 & 0.003 & 0.003 \\
\hline & $\mathrm{N}$ & 60 & 60 & 60 & 60 & 60 & 60 & 60 & 60 & 60 & 60 \\
\hline \multirow{3}{*}{ M5 } & $\begin{array}{c}\text { Pearson Correlation } \\
\text { Corelaţia Pearson }\end{array}$ & -0.190 & -0.217 & -0.160 & 0.166 & $0.265^{*}$ & $0.415^{* *}$ & 1 & -0.084 & 0.163 & 0.244 \\
\hline & Sig. (2-tailed) & 0.145 & 0.095 & 0.221 & 0.205 & 0.040 & 0.001 & & 0.523 & 0.213 & 0.061 \\
\hline & $\mathrm{N}$ & 60 & 60 & 60 & 60 & 60 & 60 & 60 & 60 & 60 & 60 \\
\hline \multirow{3}{*}{$\mathrm{MF}$} & $\begin{array}{l}\text { Pearson Correlation } \\
\text { Corelaţia Pearson }\end{array}$ & -0.153 & $0.635^{* *}$ & $-0.266^{*}$ & $-0.324^{*}$ & -0.219 & -0.127 & -0.084 & 1 & $-0.319^{*}$ & -0.085 \\
\hline & Sig. (2-tailed) & 0.244 & 0.000 & 0.040 & 0.011 & 0.093 & 0.332 & 0.523 & & 0.013 & 0.517 \\
\hline & $\mathrm{N}$ & 60 & 60 & 60 & 60 & 60 & 60 & 60 & 60 & 60 & 60 \\
\hline \multirow{3}{*}{$\mathrm{LH}$} & $\begin{array}{c}\text { Pearson Correlation } \\
\text { Corelaţia Pearson }\end{array}$ & $0.417^{* *}$ & $-0.456^{* *}$ & $0.405^{* *}$ & $0.572^{* *}$ & $0.661^{* *}$ & $0.373^{* *}$ & 0.163 & $-0.319^{*}$ & 1 & $0.768^{* *}$ \\
\hline & Sig. (2-tailed) & 0.001 & 0.000 & 0.001 & 0.000 & 0.000 & 0.003 & 0.213 & 0.013 & & 0.000 \\
\hline & $\mathrm{N}$ & 60 & 60 & 60 & 60 & 60 & 60 & 60 & 60 & 60 & 60 \\
\hline \multirow{3}{*}{$\mathrm{MH}$} & $\begin{array}{l}\text { Pearson Correlation } \\
\text { Corelaţia Pearson }\end{array}$ & 0.172 & $-0.365^{* *}$ & 0.225 & $0.450^{* *}$ & $0.534^{* *}$ & $0.371^{* *}$ & 0.244 & -0.085 & $0.768^{* *}$ & 1 \\
\hline & Sig. (2-tailed) & 0.188 & 0.004 & 0.084 & 0.000 & 0.000 & 0.003 & 0.061 & 0.517 & 0.000 & \\
\hline & $\mathrm{N}$ & 60 & 60 & 60 & 60 & 60 & 60 & 60 & 60 & 60 & 60 \\
\hline
\end{tabular}

** Correlation is significant at the 0.01 level (2-tailed).

** Corelaţia este semnificativă la nivelul 0.01 (2-tailed).

* Correlation is significant at the 0.05 level (2-tailed).

* Corelaţia este semnificativă la nivelul 0.05 (2-tailed). 
If by analyzing the Pearson correlation coefficient, a value closer to +1 is obtained, it means it is a stronger positive correlation, a value closer to -1 , a stronger negative correlation, and 0 means no correlation. Considering the significant positive and negative correlation between parameters, one can highlight that some pressure areas are directly proportional to others (like for example T1 and T2-5 with M1, M2, M4, MF) and other pressure areas are indirectly proportional to others (like for example LH and T2-5). An important result is the negative correlation between toe area and the third and fifth metatarsals bone head.

\section{CONCLUSIONS}

The present study approaches the main differences of plantar pressures in the case of young adult women, depending on the foot type and foot area analyzed. The findings of the Pearson correlation analysis demonstrate significant positive and negative correlation between foot plantar pressure parameters. Therefore, when creating the footwear bottom components, the designer should use different materials, structures and design in order to redistribute the plantar pressures according to areas of the foot.
Dacă prin analiza coeficientului de corelaţie Pearson se obţine o valoare mai apropiată de +1 atunci este o corelaţie puternic pozitivă, iar o valoare mai apropiată de -1 reprezintă o corelaţie puternic negativă, 0 înseamnă că nu există nicio corelaţie. Având în vedere corelaţiile semnificativ pozitive şi negative dintre parametri, se poate demonstra că unele zone de presiune sunt direct proporţionale $\mathrm{cu}$ altele (de exemplu T1 şi T2-5 cu M1, M2, M4, MF), iar unele zone de presiune sunt indirect proporţionale cu altele (de exemplu LH cu T2-5). Un rezultat important este corelaţia negativă dintre zona degetelor şi al treilea şi al cincilea metatarsian.

\section{CONCLUZII}

Studiul de faţă abordează diferenţele principale ale presiunilor plantare în cazul femeilor tinere adulte, în funcţie de tipul şi de zona piciorului analizat. În cadrul acestui studiu s-a arătat că există diferenţe de presiune la persoanele tinere de sex feminin, în funcţie de zona piciorului şi de tipul piciorului analizat. Rezultatele analizei Pearson demonstrează corelaţia pozitivă şi negativă semnificativă între parametrii de presiune plantară ai piciorului. În consecinţă, în momentul proiectării ansamblului inferior al încălţămintei, proiectantul trebuie să aibă în vedere echilibrarea distribuţiei presiunilor plantare pe zonele piciorului, prin forma şi structura modelului şi prin tipul materialelor folosite.

\section{REFERENCES}

1. Zheng, S., Huang, K., Tan, T., Evaluation framework on translation-invariant representation for cumulative foot pressure image, The 18th IEEE International Conference on Image Processing (ICIP), 2011, 201-204.

2. Zheng, S., Huang, K., Tan, T., Tao, D., A cascade fusion scheme for gait and cumulative foot pressure image recognition, Pattern Recogn, 2012, 45, 3603-3610, available at www.elsevier.com/locate/pr.

3. Felzenszwalb, P.F., Girshick, R.B., McAllester, D., Ramanan, D., Object detection with discriminatively trained partbased models, IEEE Trans Pattern Anal Mach Intell, 2010, 32, 1627-1645.

4. Deselnicu, D.C., Vasilescu, A.M., Mihai, A., Purcarea, A.A., Militaru, G., New products development through customized design based on customers' needs. Part 2: Foot Pathology Manufacturing Parameters, The 9th International Conference Interdisciplinarity in Engineering, INTER-ENG 2015, Procedia Technology, 2016, 22, 10591065, available at www.sciencedirect.com.

5. Sarghie, B., Mihai, A., Herghiligiu, I.V., E-learning application for 3D modelling of custom shoe lasts using templates, 
The 12th International Scientific Conference eLearning and Software for Education, Bucharest, April 21-22, 2016, 10.12753/2066-026X-16-260, p.553.

6. Herghiligiu, I.V., Mihai, A., Sarghie, B., Souto Bizarro, R., Arias, C., Framework of the e-learning training program on corporate social responsibility, The 12th International Scientific Conference eLearning and Software for Education, Bucharest, April 21-22, 2016, 10.12753/2066-026X-16-255, p. 526.

7. Ionesi, D., Ciobanu, L., Sarghie, B., E-Learning Application for a better understanding of shoes 3D modeling, 10th International Scientific Conference ELSE, ISSN 2360-2198, 2014, 10.12753/2066 026X 14 285, p.196.

8. Tao, D., Li, X., Wu, X., Maybank, S.J., General tensor discriminant analysis and gabor features for gait recognition, IEEE Trans Pattern Anal Mach Intell, 2007, 29, 10, 1700-1715.

9. Geng, X., Smith-Miles, K., Wang, L., Li, M., Wu, Q., Context-aware fusion: a case study on fusion of gait and face for human identification in video, Pattern Recogn, 2010, 43, 3660-3673.

10. He, M., Horng, S., Fan, P., Performance evaluation of score level fusion in multimodal biometric systems, Pattern Recogn, 2010, 43, 1789-1800.

11. Chang, W.R., Matz, S., Chang, C.-C., A comparison of required coefficient of friction for both feet in level walking, Safety Sci, 2012, 50, 240-243, available at www.elsevier.com/locate/ssci.

12. Gravano, S., Ivanenko, Y.P., Maccioni, G., Macellari, V., Poppele, R.E., Lacquaniti, F., A novel approach to mechanical foot stimulation during human locomotion under body weight support, Hum Mov Sci, 2011, 30, 352-367, available at www.elsevier.com/locate/humov.

13. Wrbaskic, N., Dowling, J.J., An investigation into the deformable characteristics of the human foot using fluoroscopic imaging, Clin Biomech, 2007, 22, 230-238, available at www.elsevier.com/locate/clinbiomech.

14. Păştină, M., Mihai, A., Bilalis, N., Finite element analysis for insole-sole prototypes, Proceedings of "The 4th International Conference on Advanced Materials and Systems", ICAMS 2012, Bucharest, 2012, ISSN 2068-0783, 359-364.

15. http://www.rsscan.co.uk/

16. Costea, M., Vasilescu, A.M., Hortal, G., Mihai, A., Plantar footprints analysis - case study (part 2), Revista de Pielarie Incaltaminte (Leather and Footwear Journal), 2014, 14, 4, Certex Publishing House, ISSN 15834433, 243-250.

17. Mihai, A., Hortal, G., Costea, M., Plantar footprints analysis - case study (part 1), Revista de Pielarie Incaltaminte (Leather and Footwear Journal), 2013, 13, 3, Certex Publishing House, ISSN 15834433, 201-210.

18. Mihai, A., Păştină (Costea), M., Classification of foot types, based on plantar footprint, Proceedings of "The $4^{\text {th }}$ International Conference on Advanced Materials and Systems", ICAMS 2012, Bucharest, 2012, ISSN 2068-0783, 347-352.

19. https://en.wikipedia.org/wiki/Pearson_product-moment_correlation_coefficient

20. http://www.real-statistics.com/correlation/basic-concepts-correlation/

Article received/Data primirii articolului: 24.05.2016

Accepted/Acceptat la data: 13.06.2016 